\title{
Effect of spatial variability of storm on the optimal placement of best management practices (BMPs)
}

\author{
C. L. Chang • P. T. Chiueh $\cdot$ S. L. Lo
}

Received: 27 August 2006 / Accepted: 12 February 2007 / Published online: 15 March 2007

(C) Springer Science + Business Media B.V. 2007

\begin{abstract}
It is significant to design best management practices (BMPs) and determine the proper BMPs placement for the purpose that can not only satisfy the water quantity and water quality standard, but also lower the total cost of BMPs. The spatial rainfall variability can have much effect on its relative runoff and non-point source pollution (NPSP). Meantime, the optimal design and placement of BMPs would be different as well. The objective of this study was to discuss the relationship between the spatial variability of rainfall and the optimal BMPs placements. Three synthetic rainfall storms with varied spatial distributions, including uniform rainfall, downstream rainfall and upstream rainfall, were designed. WinVAST
\end{abstract}

\section{L. Chang $(\bowtie)$}

Department of Environmental Resources Management, Chia Nan University of Pharmacy \& Science,

No. 60, Erh-Jen Rd., Sec. 1, Jen-Te,

Tainan, Taiwan, Republic of China

e-mail: f89541201@ntu.edu.tw

\section{P. T. Chiueh}

Department of Environmental Management,

Tung Nan Institute of Technology,

No. 152, Sec. 3, Peishen Rd., Shenkeng,

Taipei 222, Taiwan, Republic of China

S. L. Lo

Graduate Institute of Environmental Engineering,

National Taiwan University,

Taipei 106, Taiwan, Republic of China model was applied to predict runoff and NPSP. Additionally, detention pond and swale were selected for being structural BMPs. Scatter search was applied to find the optimal BMPs placement. The results show that mostly the total cost of BMPs is higher in downstream rainfall than in upstream rainfall or uniform rainfall. Moreover, the cost of detention pond is much higher than swale. Thus, even though detention pond has larger efficiency for lowering peak flow and pollutant exports, it is not always the determined set in each subbasin.

Keywords BMPs · NPSP · Rainfall · Spatial variation Watershed management $\cdot$ WinVAST

\section{Introduction}

Non-point source pollution (NPSP) usually plays a greater role than point source pollution in a watershed (Pegram and Bath 1995). Best management practices (BMPs) can be generally categorized into structural and non-structural. Structural BMPs is frequently accomplished using structural changes in the flow transport system, such as detention ponds, wetlands, swales, etc. Non-structural BMPs refer to the practices that reduce the quantity of pollutants without any structural facility, for instances, reducing the amount of fertilizer applied, reducing the concentration of lead in gasoline, and so on (Wanielista and Yousef 
1993). Both of them are used for controlling water quantity to avoid heavy flood events and water quality to decrease possible impact on the water body in a reservoir (Mostaghimi et al. 1997). BMPs concerns not only environmental engineering and scientific technology, but also economics and management policies. It is significant to design BMPs and decide the proper BMPs placement strategies for reaching the purpose that can not only satisfy the water quantity and water quality standard, but also lower the total cost of BMPs.

NPSP are usually washed out after a rainfall storm. Rainfall characteristics, including rainfall intensity, duration and rainfall distributions, etc. can seriously affect the export amount of NPSP. Incomplete understanding of the spatial variability of rainfall can result in much uncertainty on the calculation of runoff and water quality (Vicente 1996). Even though the spatial variation of rainfall is in a small scale, it can still have much effect on its relative runoff and NPSP (Faures et al. 1995). Meantime, the design and placement of BMPs would be different as well. The objective of this study was to discuss the relationship between the spatial variation of rainfall and the optimal BMPs placements. The analysis results can be useful for water resources and watershed management.

\section{Materials and methods}

\section{WinVAST model}

In the present study, the WinVAST model was applied to predict runoff and NPSP. WinVAST is a collection of computer programs developed for simulating stormwater runoff, NPSP and pollutant transportation. It is an event-oriented model that can be applied to multiple catchment basins. WinVAST combines widely used techniques, which involve calculating rainfall abstractions; generating overland flow hygrographs; estimating flood routing from upstream subbasins to downstream subbasins; computing NPSP wash-off from subbasins; simulating routing pollutants, and predicting the condition through selected BMPs. WinVAST treats multi-catchment basins as a treeview structure, so a complex representation of the entire catchment basin can be easily generated (Tisdale et al. 1996; Yu et al. 2003a).
Runoff simulation The phenomenon of abstraction, depression, infiltration, and so on, can be simulated in WinVAST model. The necessary information for modeling involves rainfall data and watershed condition. Firstly, the stream distribution and subbasin divisions should be defined by a tree-view structure. It should describe the relationship between the upstream basins and downstream basins. Model users can decide the numbers of subbasins in a watershed in accordance with their accepted accuracy of simulation results. The rainfall characteristic, land-use condition, soil type, stream length, etc. would be different in each subbasin. Through the subbasin division, different input parameters based on various hydrology algorithms can be applied in each subbasin. Meantime, the simulation time, interval time and system unit should be determined.

The required data for each subbasin includes base flow, subbasin area, average slope, stream length, rainfall abstraction and infiltration, unit hydrograph, and so on. WinVAST provides three methods for inputting rainfall data, three methods for calculating rainfall abstraction, and four types of unit hydrograph for evaluating the relation between rainfall and runoff. Moreover, the flood routing is also significant in runoff simulation. The Muskingum method is applied to calculate flood routing in WinVAST model. The major parameters in the Muskingum method is the weight factor, $X$, and routing constant, $K$.

NPSP simulation and BMPs calculation WinVAST model integrates runoff and NPSP simulation in a single interface. The calculation of predicting pollutant transportation and decay process is similar to the algorithm of STORM (Storage, Treatment, Overflow, Runoff Model) developed by the US Army Corps of Engineers. NPSP is always washed out after a rainfall storm. The pollutant accumulation on the land surface prior to a storm event is computed by the following formula:

$P(p, l)=\operatorname{AREA}(l) * \operatorname{LR}(p, l) * \mathrm{DD}+P 0(p, l)$

where $P(p, l)$ is the accumulation of pollutant $p$ on land use $l$ just prior to storm event; AREA $(l)$ is the area of land use $l$; LR $(p, l)$ is the loading rate for 
pollutant $p$ on land use $l$; DD is the number of dry days since the previous storm; $P 0(p, 1)$ is the weight of pollutant $p$ on land use $l$ just after the previous storm.

Moreover, the pollutant wash-off from the land surface during a storm event can be computed by the following general formula:

$$
\begin{aligned}
M(p, l)= & A(p) * P(p, l) *(1.0-\exp [-k(p) * R]) \\
& +\operatorname{FSUS}(p) * M(\operatorname{sus})+\operatorname{FSET}(p) * M(\text { set })
\end{aligned}
$$

where $M(p, 1)$ is the wash-off rate of pollutant $p$ from land use $l ; A(p)$ is the fraction of pollutant $p$ available for wash-off from the land surface; $K(p)$ is wash-off decay coefficient for pollutant $p ; R$ is surface runoff; FSUS $(p)$ is the fraction of suspended solids that is pollutant $p ; M$ (sus) is the wash-off rate of suspended solids; FSET $(p)$ is the fraction of settleable solids that is pollutant $p ; M$ (set) is the wash-off rate of settleable solids. Moreover, WinVAST model provides the calculation for two types of BMPs, including detention pond and swale. Based on the design of detention pond and swale, the amount of infiltration and removed pollutant can be determined.

\section{Scatter search}

The meta-heuristic search techniques, such as genetic algorithm (GA) and scatter search, are much less restrictive in problem formulation and can be used for complex systems. GA is the most commonly used techniques in the field of hydrological and environmental engineering at the present time (Wang 1991; Cheng et al. 2002). It uses stochastic search procedures based on probabilistic rather than deterministic search rules. Scatter search shares some commonalties with GA, although it also has a number of quite distinct features. On the other hand, GA is based on the idea of choosing parents randomly to produce offspring, and further on introducing randomization to determine which components of the parents should be combined. Contrarily, scatter search does not emphasize randomization, but incorporate strategic responses that take account of evaluation and history (Yu et al. 2003b).
Due to this feature of scatter search, it is expected that scatter search can find the near-optimal solution in a more efficient way, particularly in optimization problems of complex systems (Glover 1999). Moreover, scatter search is an evolutionary algorithm that constructs solutions by combining other. The purpose of this method is to enable the implementation of solution procedures that can generate new solutions from combined elements. Recent studies demonstrate the practical advantages of this approach for solving a diverse array of optimization problems (Glover 1977, 1998; Campos et al. 1999). Therefore, the extra computer program of WinVAST model based on the algorithms of scatter search for the optimization of BMPs placement was applied in this work. The basic procedure of scatter search includes: (1) Diversification generation method, which is to generate a collection of diverse trial solutions as initial inputs; (2) Improvement method, which is to enhance the trial solutions; (3) Reference set update method, which is to build a reference set according to the quality of solutions; (4) Subset generation method, which is to produce a subset of its solutions as a basis for creating combined solutions; and (5) Solution combination method, which is to transform a subset of solutions into combined solution vectors.

Problem formulation

Limited actual data would be an obstacle to developing or applying models. Thus, synthetic data, which can overcome the restriction due to inadequate measured data (Hromadka 1996a, b), was created in this study. Figure 1 presents the synthetic case area designed in this work. The case area has 10 subbasins. Each of which has an area of $2 \mathrm{~km}^{2}$. The length of the main stream in each subbasin is $2000 \mathrm{~m}$. All of the land is used as forest, and the average slope is $5 \%$. The curve number $(\mathrm{CN})$ for the forest is 70 . The general pollutant loading of SS in forest is $0.15 \mathrm{~kg} / \mathrm{ha} /$ day. Three rainfall storms with varied spatial distributions, including uniform rainfall, downstream rainfall and upstream rainfall, were designed. The total precipitations of these three rainfall storms were all the same in the case area. The average rainfall depth was $4 \mathrm{~mm}$, and rainfall duration was $5 \mathrm{~h}$. The precipitation standard deviation of each subbasin is zero when the rainfall is uniform. In this study, the precipitation 


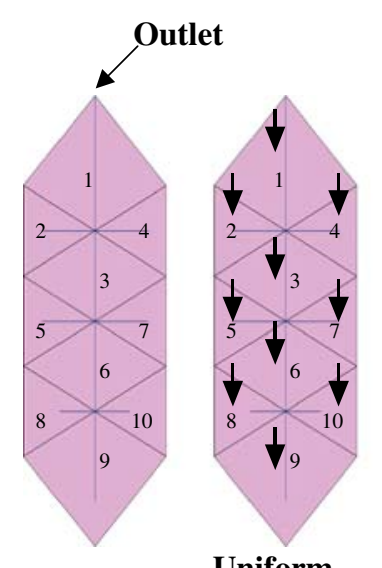

\section{Uniform Upstream \\ Rainfall}
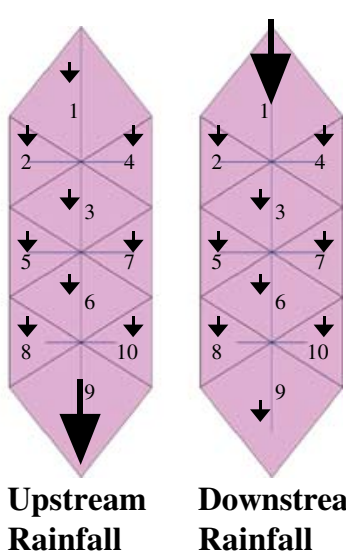

Downstream Rainfall

Fig. 1 Synthetic case area and the illustration of rainfall storms with various spatial distributions

standard deviation of each subbasin was given by $3.2 \mathrm{~mm}$ to represent the spatial rainfall variability. The upstream rainfall exhibits that the heavy rainfall focuses in the upstream subbasin, subbasin 9. On the contrary, the downstream rainfall displays that the heavy rainfall is close to the outlet, subbasin 1.

Detention pond and swale were selected for being structural BMPs in this work. There were four choices

of BMPs placement in each subbasin, including none BMPs, only detention pond, only swale, and both of detention pond and swale. Thus, the total number of possible BMPs placement in the case area was $4^{10}$. The design of detention pond and swale was fixed in this study. Restated, the parameters in WinVAST model for representing the type and scale of detention pond or swale were constant. The "Weir-Pipe equation" was applied for calculating the efficiency of detention pond. The weir height was designed as $1.2 \mathrm{~m}$. The average pond area was about $0.02 \mathrm{~km}^{2}$. The length of swale was designed as $100 \mathrm{~m}$. The manning coefficient was 0.2 . The infiltration rate was $7.5 \mathrm{~cm} / \mathrm{h}$. The swale slope was about $2 \%$. The BMPs placement is determined based on the objective function defined as follows:

$$
\begin{aligned}
& \text { Objective function: } \operatorname{Min} \sum_{i=1}^{10} \alpha \operatorname{COST}\left(\mathrm{dp}_{i}\right) \\
& \quad+\beta \operatorname{COST}\left(\mathrm{sw}_{i}\right)
\end{aligned}
$$

where $\alpha$ and $\beta$ are binary variable. When the detention pond or the swale are set, the value of $\alpha$ or $\beta$ is given by 1 ; contrarily, the value is given by 0 . $\operatorname{COST}\left(\mathrm{dp}_{i}\right)$ is the cost of detention pond in subbasin $i$,

\begin{tabular}{|c|c|c|c|c|c|c|}
\hline Constraint & $\mathrm{R}$ & 0.5 & 0.6 & 0.7 & 0.8 & 0.9 \\
\hline \multirow[t]{5}{*}{ Uniform rainfall } & Min $\mathrm{c}$ & 552701 & 552701 & 552701 & 605974 & 616628 \\
\hline & $\mathrm{R} \_\mathrm{m}$ & 0.76 & 0.76 & 0.76 & 0.90 & 0.90 \\
\hline & D_Qp & 0.29 & 0.29 & 0.29 & 0.29 & 0.29 \\
\hline & BMPs & 100000 & 10000 & $\begin{array}{llllll}1 & 0 & 0 & 0 & 0\end{array}$ & $\begin{array}{llllll}3 & 0 & 0 & 0 & 0\end{array}$ & 30203 \\
\hline & placement & $\begin{array}{llllll}0 & 0 & 0 & 0 & 0\end{array}$ & $\begin{array}{llllll}0 & 0 & 0 & 0 & 0\end{array}$ & $\begin{array}{llllll}0 & 0 & 0 & 0 & 0\end{array}$ & 22022 & 00222 \\
\hline \multirow[t]{5}{*}{ Upstreamrainfall } & Min_c & 552701 & 563355 & 563355 & 584664 & 584664 \\
\hline & R_m & 0.76 & 0.76 & 0.76 & 0.90 & 0.90 \\
\hline & D_Qp & 0.29 & 0.29 & 0.29 & 0.29 & 0.29 \\
\hline & BMPs & 10000 & 12000 & 10020 & 32000 & 30200 \\
\hline & placement & $\begin{array}{llllll}0 & 0 & 0 & 0 & 0\end{array}$ & $\begin{array}{llllll}0 & 0 & 0 & 0 & 0\end{array}$ & $\begin{array}{llllll}0 & 0 & 0 & 0 & 0\end{array}$ & 000002 & 020000 \\
\hline \multirow[t]{5}{*}{ Downstreamrainfall } & Min_c & 563355 & 574010 & 552701 & 627283 & 1148020 \\
\hline & R_m & 0.76 & 0.61 & 0.76 & 0.81 & 0.90 \\
\hline & D_Qp & 0.29 & 0.22 & 0.29 & 0.29 & 0.29 \\
\hline & BMPs & 10000 & 00300 & 10000 & 12220 & 12120 \\
\hline & placement & 000002 & 00002 & $\begin{array}{llllll}0 & 0 & 0 & 0 & 0\end{array}$ & 22022 & 20002 \\
\hline
\end{tabular}

Table 1 The minimum BMPs cost and the optimal BMPs placement in these three rainfall storms with different spatial distribution

Min_C is minimum BMPs cost (Unit: *\$)

$R \_m$ is TSS removal rate in minimum BMPs cost

$R$ is TSS removal rate (constraint)

$D \_Q p$ is the decrease rate of peak flow 
which is assessed by the volume of detention pond. $\operatorname{COST}\left(\mathrm{sw}_{i}\right)$ is the cost of swale in subbasin $i$, which is calculated by the area of swale. Equations 4 and 5 show the calculation of the cost of detention pond and the cost of swale respectively, i.e. $\operatorname{COST}\left(\mathrm{dp}_{i}\right)$ and $\operatorname{COST}\left(\mathrm{sw}_{i}\right)$.

$\operatorname{COST}\left(\mathrm{dp}_{i}\right)=K_{1} * 18.5 * V^{0.7}$

(Brown and Schueler 1997)

$$
\operatorname{COST}\left(\mathrm{sw}_{i}\right)=K_{2} * 1850 * A
$$

(Schueler 1987)

where $K_{1}$ and $K_{2}$ are inflation rate and given by 1.74 and 2.4 respectively. $V$ is the volume of detention pond in the unit of $\mathrm{ft}^{3} . A$ is the area of swale in the unit of acre. The objective of the optimal BMPs placement study was to determine locations of detention ponds and swales in order to minimize the total cost of BMPs while satisfying the TSS removal rate of 50, 60, 70, 80 and 90\% separately. Also, the BMPs placement should be arranged in a feasible range.

\section{Results and discussion}

In this work, the design of detention pond and swale was constant. Thus, when the total numbers of detention pond and swale are fixed, the BMPs cost is the same as well, even though the BMPs placement and TSS removal rate can be different. The results show that spatial rainfall variability can greatly affect runoff volumes, peak flow, arrival time, and pollutant export. Therefore, the optimal placement of BMPs can be rather different for these three rainfall storms with varied spatial distribution. The total cost of BMPs in the optimal placement is different as well. As shown in Table 1, when the restriction of TSS removal rate is large, e.g. 0.8 and 0.9 , the total cost of BMPs is the highest in downstream rainfall, secondly in uniform rainfall and the lowest in upstream rainfall. When the restriction of TSS removal rate is $0.5,0.6$ or 0.7 . Mostly, only one detention pond or swale can efficiently lower the pollutant exports, and satisfy the constraints of TSS removal rate. Thus, the differences of BMPs placement and the minimum BMPs cost between each spatial rainfall distribution are not noticeable.
Table 2 shows the set location of BMPs and its relation to the decrease rate of peak flow and TSS removal rate. The results exhibit that the detention pond has more efficiency for decreasing the peak flow and lowering the TSS export at the outlet than the swale does. However, one detention pond costs 552,701 dollars, but one swale only costs 10,655 dollars. The cost of detention pond is even over 50 times higher than it of swale. Therefore, although detention pond can control the peak flow and pollutant export much better, it is not supposed to be the inevitable optimal BMPs determination for each subbasin based on the objective to minimize the total cost of BMPs. If the swales are set in each subbasin, it costs 106,550 dollars, which is less than the cost of one detention pond. However, the total TSS removal rate at the outlet is only about $35 \%$, when setting swales in each subbasin. Since the TSS removal rate in this study was asked to be higher than $50 \%$ anyhow, the optimization process certainly selected one detention pond rather than a group of swales.

Scatter search is the major tool for optimizing the BMPs placement. In this study, the run time was given by 1,000 . The convergence of optimal results is

Table 2 The relation between set location of BMPs and its relative decrease rate of peak flow and TSS removal rate

\begin{tabular}{llll}
\hline $\begin{array}{l}\text { Set } \\
\text { location }\end{array}$ & BMPs & $\begin{array}{l}\text { Q decrease } \\
\text { rate }\end{array}$ & $\begin{array}{l}\text { TSS removal } \\
\text { rate }\end{array}$ \\
\hline subbasin1 & Detention pond & 0.29 & 0.76 \\
& Swale & 0.00 & 0.10 \\
subbasin2 & Detention pond & 0.14 & 0.10 \\
& Swale & 0.00 & 0.02 \\
subbasin3 & Detention pond & 0.22 & 0.53 \\
& Swale & 0.00 & 0.07 \\
subbasin4 & Detention pond & 0.14 & 0.10 \\
& Swale & 0.00 & 0.02 \\
subbasin5 & Detention pond & 0.11 & 0.10 \\
& Swale & 0.00 & 0.02 \\
subbasin6 & Detention pond & 0.24 & 0.31 \\
& Swale & 0.00 & 0.04 \\
subbasin7 & Detention pond & 0.11 & 0.10 \\
& Swale & 0.00 & 0.02 \\
subbasin8 & Detention pond & 0.06 & 0.10 \\
& Swale & 0.00 & 0.02 \\
subbasin9 & Detention pond & 0.06 & 0.10 \\
& Swale & 0.00 & 0.02 \\
subbasin10 & Detention pond & 0.06 & 0.10 \\
& Swale & 0.00 & 0.02 \\
\hline & & &
\end{tabular}


not always noticeable. Consequently, the searching results are probably not the best. However, the nearoptimal results are still significant. If the accurately optimal results are required, the run time has to be increased. It is not usually practicable to use an average precipitation to represent the rainfall in a watershed. If the spatial variation of rainfall is serious, it will be essential to consider the actual rainfall distribution. The analysis faults can result in unnecessarily excess cost for BMPs design and placements. Moreover, the pollutant concentration would exceed the water quality standard, even though the total cost of BMPs is high. Therefore, it is very important to realize the spatial rainfall variability in a watershed for adequately predicting runoff and NPSP, and for the basis of water resource and watershed management strategies.

\section{Conclusions}

The spatial rainfall variability can affect the runoff volume, arrival time of peak flow, and pollutant export. The design and placement of BMPs can be influenced as well. The present paper discusses the relationship between the spatial variation of rainfall and the optimal BMPs placement. The results are concluded as follows:

1. The design of BMPs is constant herein. Thus, when the total numbers of detention pond and swale are fixed, the minimum total cost of BMPs is the same, even though the BMPs placement is different in a watershed.

2. When the restriction of TSS removal rate is large, mostly the total cost of BMPs is higher in downstream rainfall than in upstream rainfall or uniform rainfall.

3. The detention pond has larger efficiency for lowing the peak flow and pollutant export. However, the cost of detention pond is much higher than it of swale, so detention pond is not always determined for setting in all the subbasins in order to minimize BMPs cost.

4. Scatter search can only find near-optimal results. If the accurately optimal results are needed, the run time has to be increased.
In future studies, the restrictions in problem formulation can be adjusted depending on the water quality or water quantity standard. They are essential factors, which may influence the optimal BMPs placement. Moreover, the design of BMPs can be also optimized. This work demonstrated that the spatial variability of rainfall have serious effect on the optimal BMPs placement. Economically optimal design and placement of BMPs can avoid unnecessary cost of BMPs and also observe all the water quality or water quantity standard.

Acknowledgement The authors would like to thank the National Science Council of the Republic of China for financially supporting this research under Contract no. NSC93-2211-E-002-030.

\section{References}

Brown, W., \& Schueler, T. (1997). National pollutant removal performance database for stormwater best management practices. Ellicott City, MD, USA: Center for Watershed Protection.

Campos, V., Laguna, M., \& Marti, R. (1999). Scatter search for the linear ordering problem. In: D. Corne, M. Dorigo, \& F. Glover (Eds.), New methods in optimization. New York: McGraw-Hill.

Cheng, C. T., Ou, C. P., \& Chau, K. W. (2002). Combining a fuzzy optimal model with a genetic algorithm to solve multi-objective rainfall-runoff model calibration. Journal of Hydrology, 268, 72-86.

Faures, J. M., Goodrich, D. C., Davis, A. W., \& Soroosh, S. (1995). Impact of small-scale spatial variability on runoff modeling. Journal of Hydrology, 173, 309-326.

Glover, F. (1977). Heuristics for integer programming using surrogate constraints. Decision Science, 8(1), 156-166.

Glover, F. (1998). A template for scatter search and path relinking. In: J. K. Hao, E. Lutton, E. Ronald, M. Schoenauer, \& D. Snyers (Eds.), Artificial evolution, lecture notes in computer science 1363 (pp. 13-54). Berlin Heidelberg New York: Springer.

Glover, F. (1999). Scatter search and path relinking. In: D. Corne, M. Dorigo, \& F. Clover (Eds.), New methods in optimization. New York: McGraw-Hill.

Hromadka, T. V. (1996a). A rainfall-runoff probabilistic simulation program: 1. Synthetic data generation. Environmental Software, 11(4), 235-242.

Hromadka, T. V. (1996b). A rainfall-runoff probabilistic simulation program: 2. Synthetic data analysis. Environmental Software, 11(4), 243-249.

Mostaghimi, S., Park, S. W., Cooke, R. A., \& Wang, S. Y. (1997). Assessment of management alternatives on a small agricultural watershed. Water Research, 31(8), 1867-1878.

Pegram, G. C., \& Bath, A. J. (1995). Role of non-point sources in the development of a water quality management plan 
for the Mgeni River Catchment. Water Science and Technology, 32, 5-6.

Schueler, T. (1987). Controlling urban runoff: A practical manual for planning and designing urban best management practices. Washington, DC, USA: Metropolitan Washington Council of Governments.

Tisdale, T. S., Kaighn, R. J., \& Yu, S. L. (1996). The Virginia storm (VAST) model for stormwater management - User's guide version 6.0. Charlottesville, VA, USA: Department of Civil Engineering, University of Virginia.

Vicente, L. L. (1996). On the effect of uncertainty in spatial distribution of rainfall on catchment modeling. Catena, 28, 107-119.
Wang, Q. J. (1991). The genetic algorithm and its application to calibrating conceptual rainfall-runoff models. Water Resources Research, 27(9), 2467-2471.

Wanielista, M. P., \& Yousef, A. Y. (1993). Stormwater management. New York: Wiley.

Yu, S. L., Stanford, R. L., \& Zhai, Y. Y. (2003a). Virginia stormwater model for windows - User's manual version 1.0. Charlottesville, VA, USA: Department of Civil Engineering, University of Virginia.

Yu, S. L., Zhen, X. Y., \& Zhai, Y. Y. (2003b). Development of a BMP placement strategy for VDOT. Charlottesville, VA, USA: Department of Civil Engineering, University of Virginia. 